\title{
LCDiXRay: a user-friendly program for powder diffraction indexing of Columnar Liquid Crystals
}

Nicolas Godbert, ${ }^{a_{\star}}$ Alessandra Crispini, ${ }^{a}$ Mauro Ghedini, ${ }^{a}$ Manuela Carini, ${ }^{b}$ Francesco Chiaravalloti, ${ }^{c}$ Andrea Ferrise $^{d}$

a Centro di Eccelenza CEMIF.CAL, LASCAMM CR-INSTM della Calabria, Dipartimento di Chimica e Tecnologie Chimiche, Università della Calabria, 87036 Arcavacata di Rende (CS), Italy.

${ }^{b}$ Dipartimento di Ingegneria per l'Ambiente ed il Territorio e Ingegneria Chimica, Università della Calabria, 87036 Arcavacata di Rende (CS), Italy.

${ }^{c}$ Dipartimento di Fisica, Università della Calabria, 87036 Arcavacata di Rende (CS), Italy.

${ }^{d}$ Dipartimento di Informatica, Modellistica, Elettronica e Sistemistica, Università della Calabria, 87036 Arcavacata di Rende (CS), Italy.

Correspondence email: nicolas.godbert@unical.it

\section{Electronic Supplementary Information}

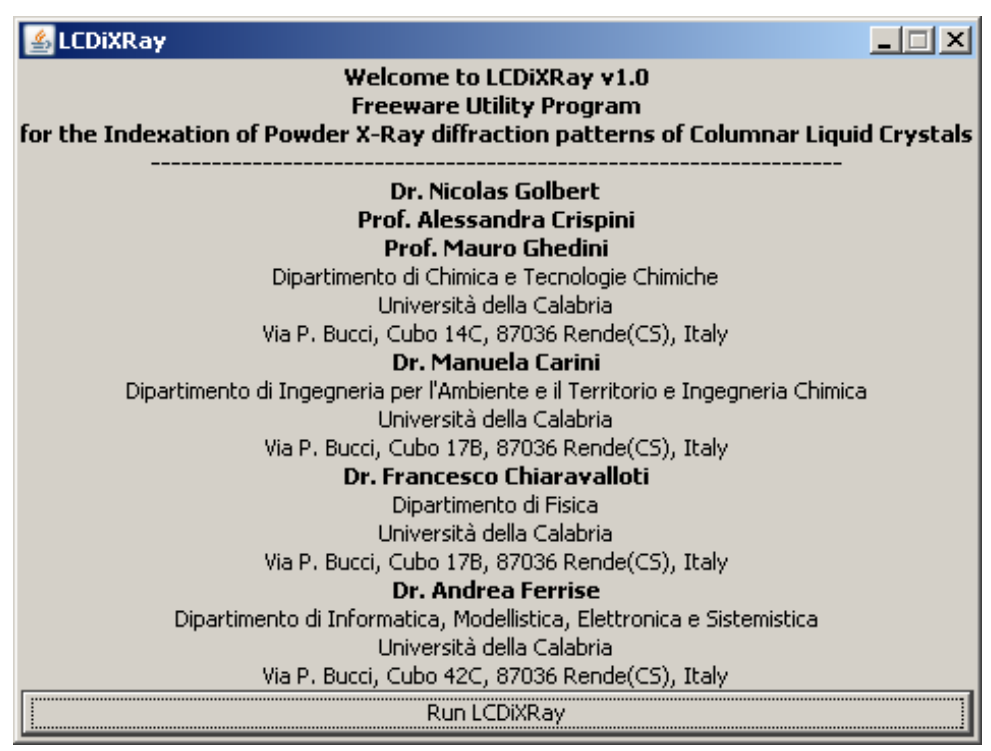

Figure S1: Presentation Window of LCDixRay (version 1.0) 


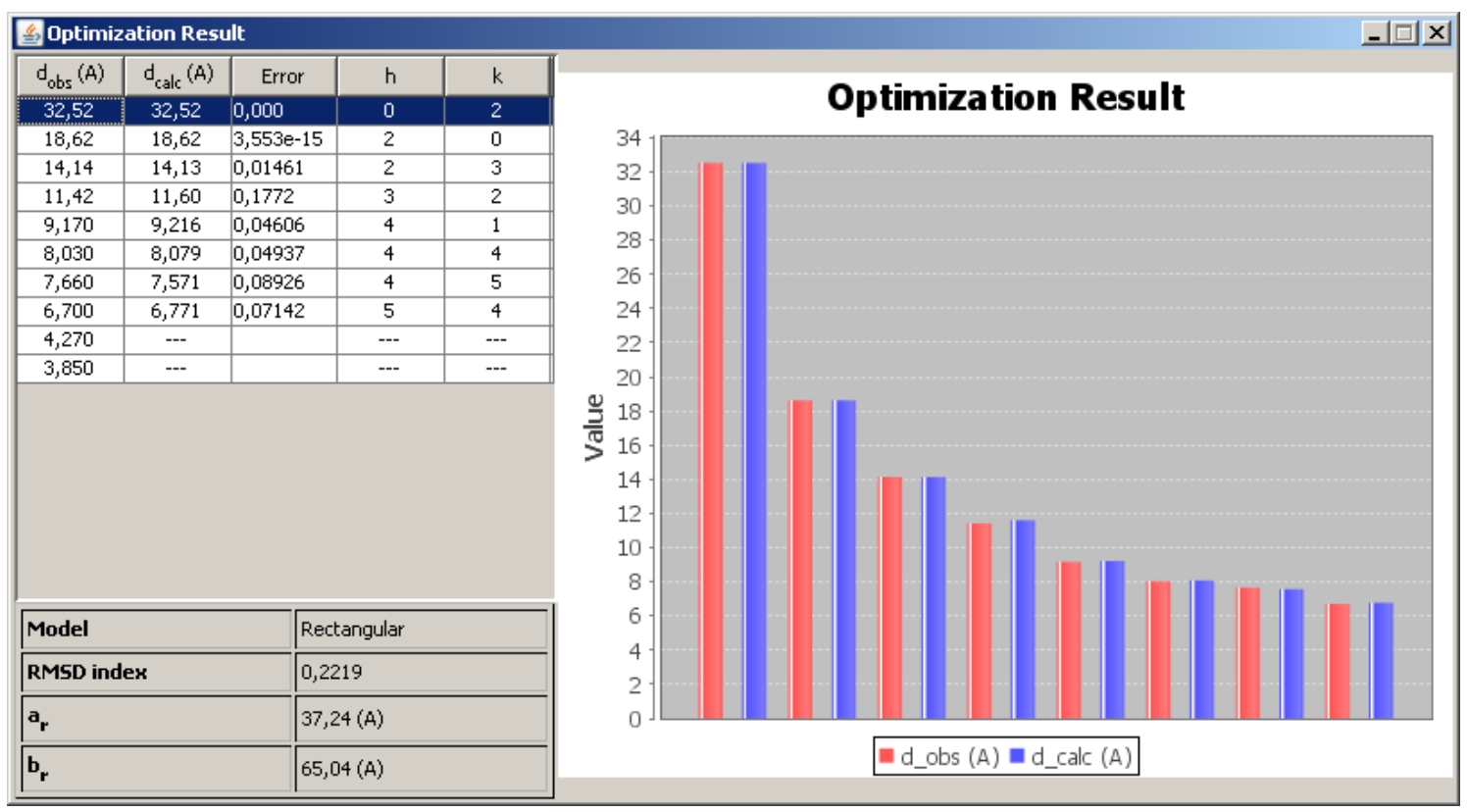

Figure S2: LCDiXRay results obtained for discotic (II) in a $\mathrm{Col}_{\mathrm{r}}$ model without refinement of data

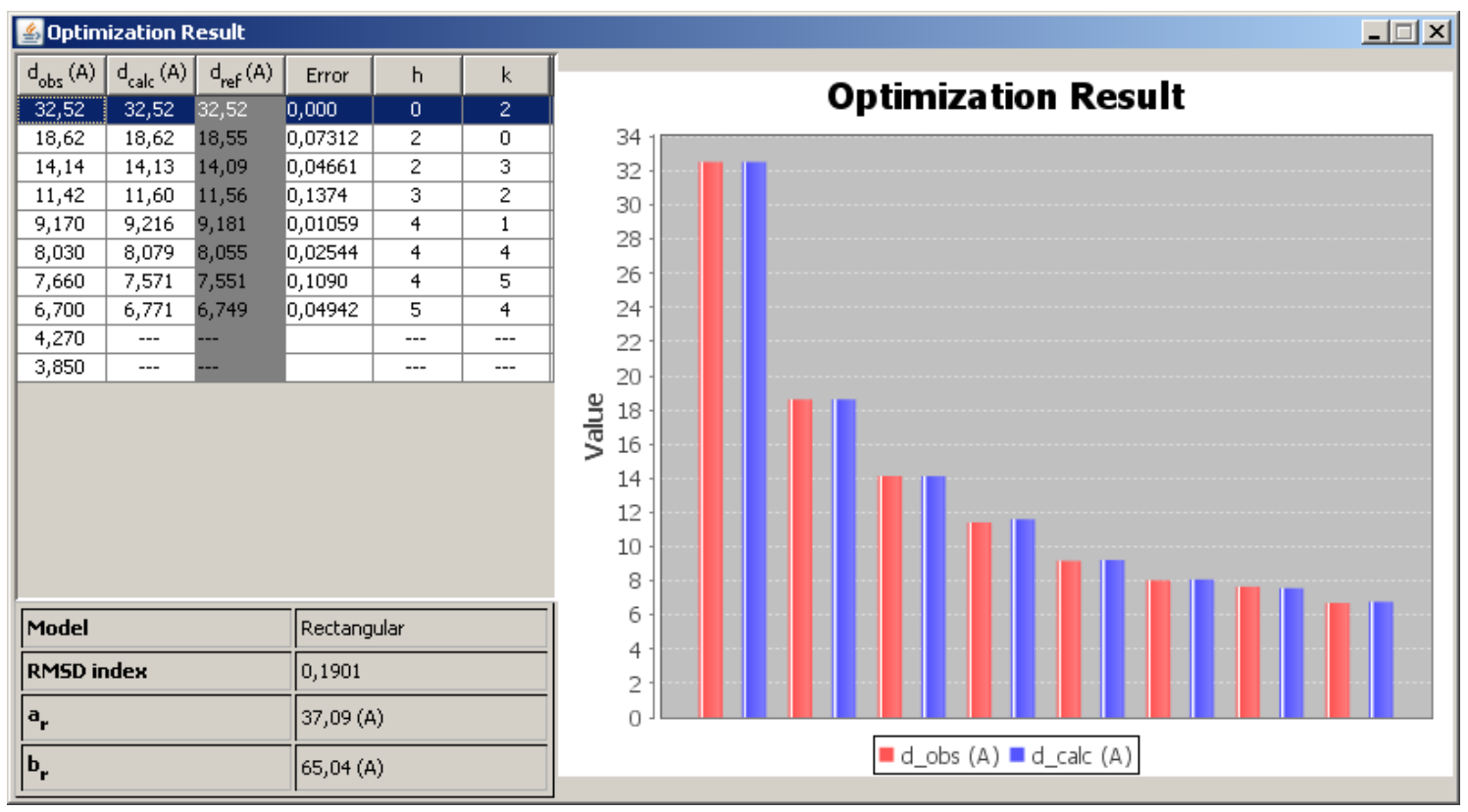

Figure S3: LCDiXRay results obtained for discotic (II) in a $\mathrm{Col}_{\mathrm{r}}$ model after refinement of data 


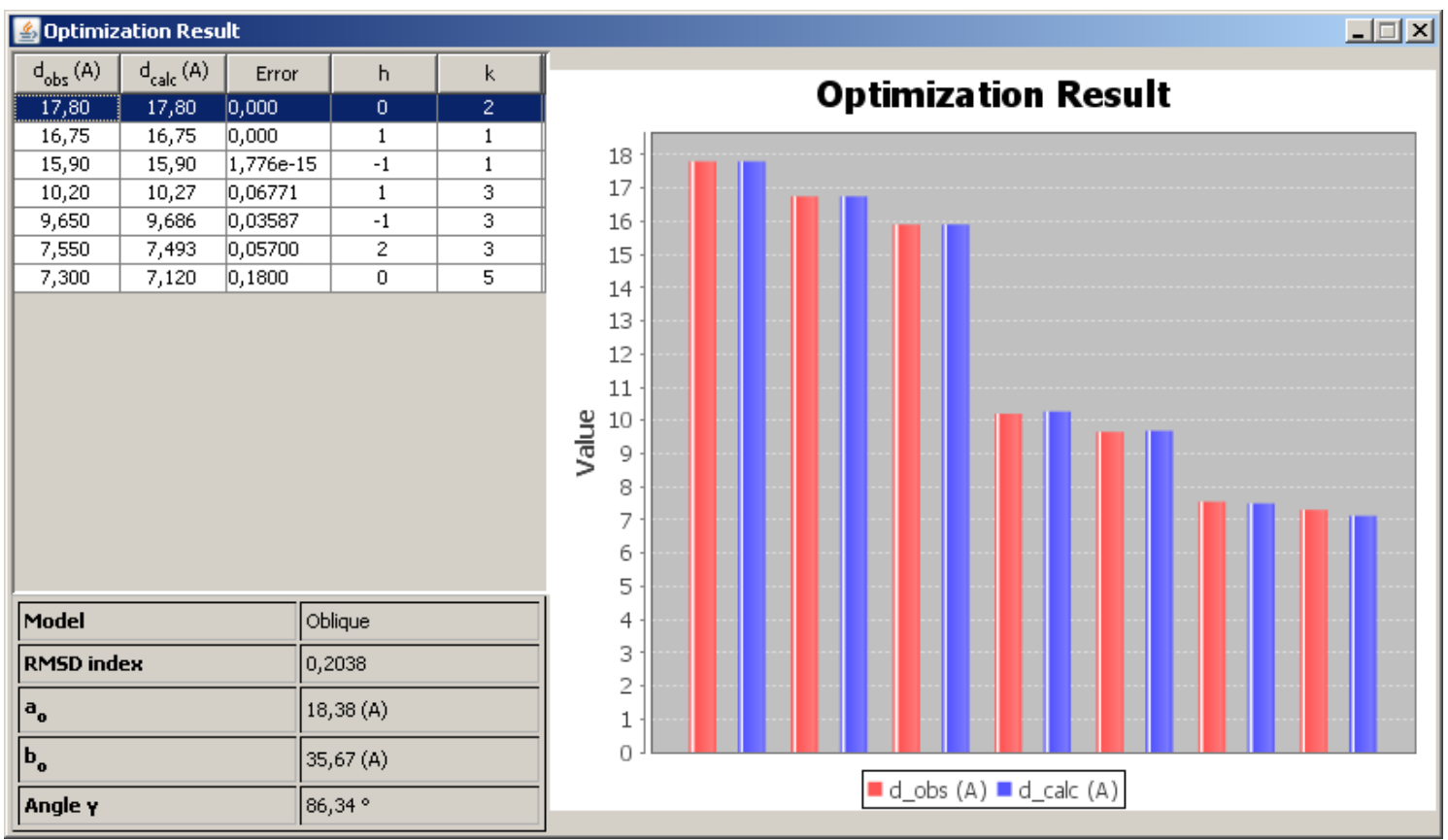

Figure S4: LCDiXRay results obtained for discotic (III) in a $\mathrm{Col}_{\mathrm{o}}$ model without refinement of data

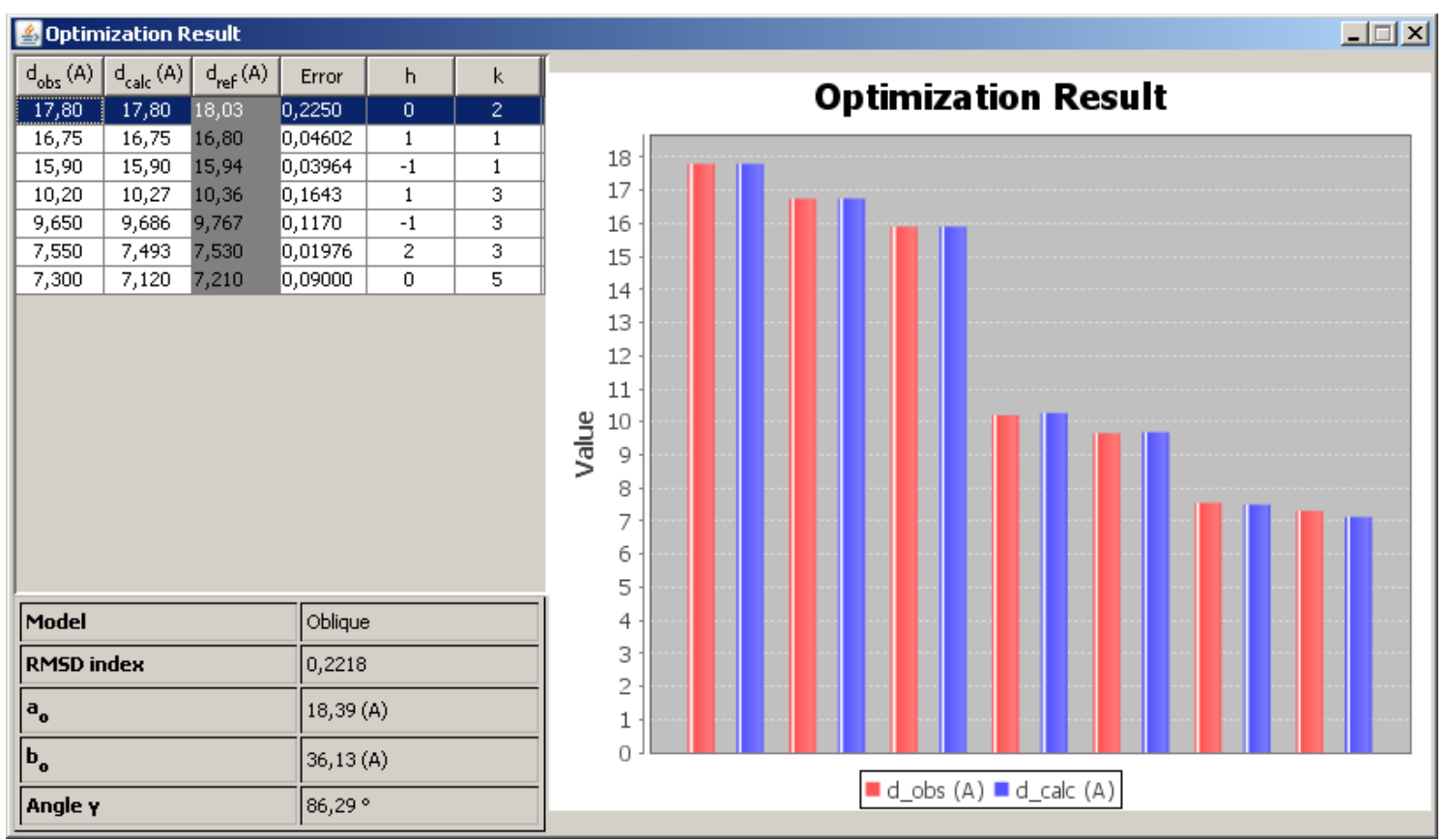

Figure S5: LCDiXRay results obtained for discotic (III) in a $\mathrm{Col}_{\mathrm{o}}$ model after refinement of data 